This item was submitted to Loughborough's Research Repository by the author.

Items in Figshare are protected by copyright, with all rights reserved, unless otherwise indicated.

\title{
Large eddy simulation of premixed combustion in spark ignited engines using a dynamic flame surface density model
}

PLEASE CITE THE PUBLISHED VERSION

http://dx.doi.org/10.4271/2013-01-1086

PUBLISHER

(C) SAE International

VERSION

AM (Accepted Manuscript)

LICENCE

CC BY-NC-ND 4.0

\section{REPOSITORY RECORD}

Ranasinghe, Chathura P., W. Malalasekera, and Andrew Clarke. 2019. "Large Eddy Simulation of Premixed Combustion in Spark Ignited Engines Using a Dynamic Flame Surface Density Model". figshare. https://hdl.handle.net/2134/14046. 
This item was submitted to Loughborough's Institutional Repository (https://dspace.lboro.ac.uk/) by the author and is made available under the following Creative Commons Licence conditions.

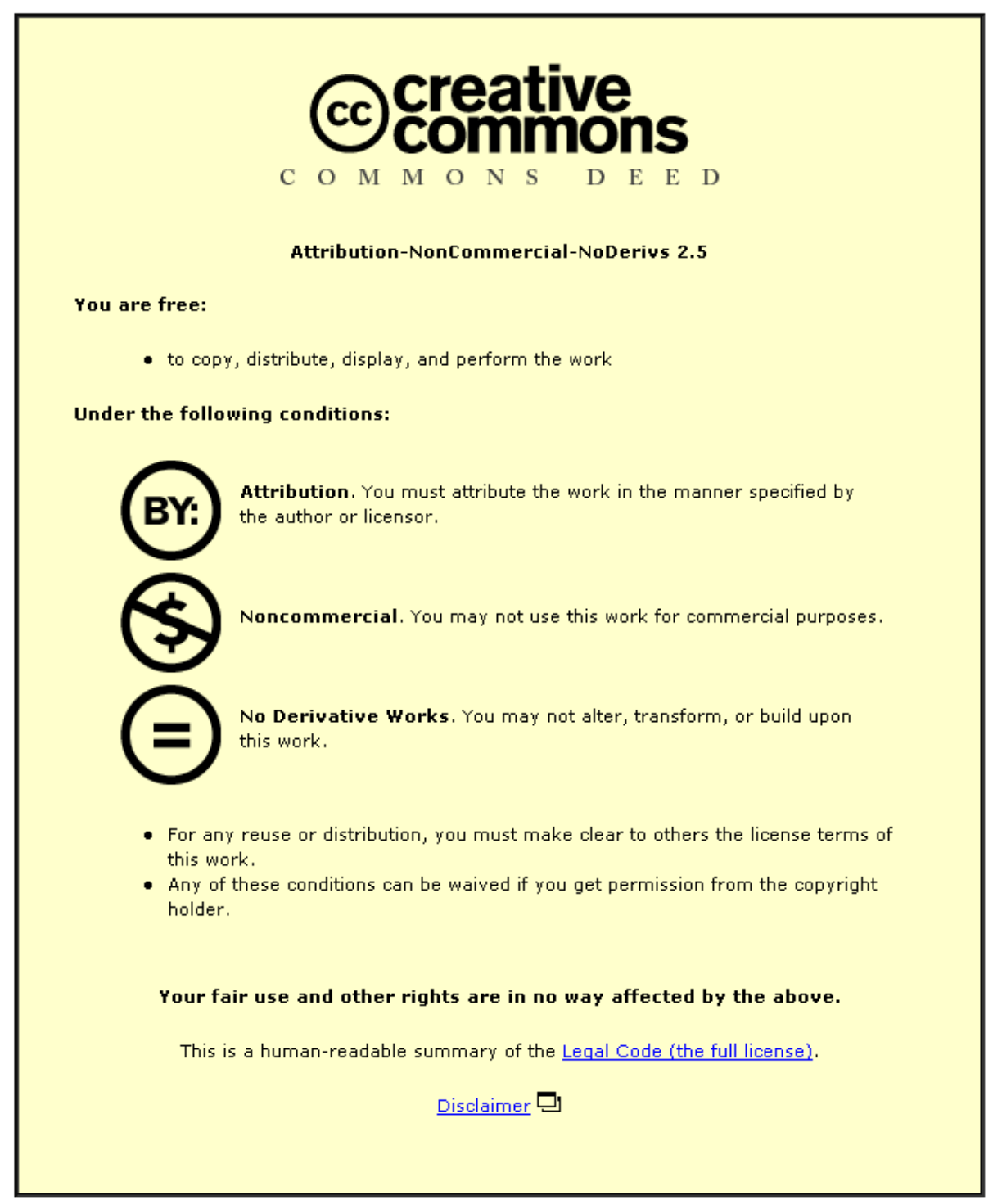

For the full text of this licence, please go to: http://creativecommons.org/licenses/by-nc-nd/2.5/ 


\section{Large Eddy Simulation of Premixed Combustion in Spark Ignited Engines Using a Dynamic Flame Surface Density Model Author, co-author (Do NOT enter this information. It will be pulled from participant tab in MyTechZone)

\author{
Affiliation (Do NOT enter this information. It will be pulled from participant tab in MyTechZone)
}

Copyright $\odot 2012$ SAE International

\section{$\underline{\text { ABSTRACT }}$}

In this work, cyclic combustion simulations of a spark ignition engine were performed using the Large Eddy Simulation techniques. The KIVA-4 RANS code was modified to incorporate the LES capability. The flame surface density approach was implemented to model the combustion process. Ignition and flame kernel models were also developed to simulate the early stage of flame propagation. A dynamic procedure was formulated where all model coefficients were locally evaluated using the resolved and test filtered flow properties during the fully developed phase of combustion. A test filtering technique was adopted to use in wall bounded systems. The developed methodology was then applied to simulate the combustion and associated unsteady effects in a spark ignition engine. The implementation was validated using the experimental data taken from the same engine.

Results show that, even with relatively coarser meshes used in this work, present LES implementation has been able to resolve the evolution of a large number of in-cylinder flow structures, which are more influential for engine performance. Predicted combustion rate and pressure rise is also in good agreement with the measurements. The limits of cyclic variations are well within the experimentally observed range. It has also been able to demonstrate the limits of cyclic fluctuations to a reasonable degree even with a fewer number of simulation cycles. A significant variation of flame propagation has also been predicted by the simulations.

\section{INTRODUCTION}

Use of the Large Eddy Simulation (LES) techniques has become increasingly popular for engineering simulations. In Reynolds Averaged Navier Stokes (RANS) method, the entire flow spectrum has to be modelled so that the predictions are model dependent to a larger extent. Whereas in LES, most of the flow field is resolved and only the sub-grid scale (SGS) part is modelled. Hence, LES results are more accurate, less model dependent and provide detailed information. In addition, LES is very much appropriate for engine simulations as it provides the opportunity to simulate inherent unsteady phenomena such as cyclic variations and combustion Page 1 of 14 instabilities. Therefore, the use of LES in engine modelling provides a more reliable way of investigating operational and geometric refinements.

LES techniques have been widely used in non-reacting flow modelling, but its application to combustion simulation is still in a preliminary stage. In particular, studies devoted to internal combustion (IC) engine simulations using LES is very limited. Studies [1-5] may be identified as some of the promising cold flow simulations which reveal the potential of LES predictions. Remarkably, compared to RANS, overall predictions of these simulations have found to be much better in agreement with experimental results. Application of LES in reacting flow modelling has largely been limited to nonpremixed combustion $[6,7]$.

Premixed combustion modelling with LES is particularly a challenging task due to a number of difficulties detailed below. Usually, the premixed laminar flame thickness $\delta_{L}$, is so thin and it cannot be resolved using the classical LES mesh sizes. For example the flame thickness in SI engine applications is about $0.1 \mathrm{~mm}$ [8]. In order to adequately resolve the flame front, a minimum of 5-10 grid points are needed with a typical finite volume based CFD code [9]. Therefore, this resolution requirement is prohibitively expensive compared to the mesh resolution possible with current computing power, which is typically about $0.5 \mathrm{~mm}$.

A possible solution is to neglect this physical consideration and model the combustion process using an eddy break-up (EBU) type formulation. In such an approach, any modelling discrepancy may be absorbed by an adjustable model constant. The EBU model has two major short comings: negligence of the chemistry interaction with combustion and poor predictions near highly strained regions such as near walls. Moreover, the EBU model constant seems to be strongly dependent on the flow condition and mesh configuration. However, there is only very little work carried out to investigate the applicability of this type of formulations [10] and some initial applications of the LES-EBU model can be found in $[11,12]$.

The $G$-equation (level-set) approach is also an alternative method where the flame front is described as a zero thickness 
surface. The propagation of the surface may be tracked by the field variable $\tilde{G}$ and the flame front is taken as the isolevel $\tilde{G}=G^{*}$. Where, $G^{*}$ is often defined to be zero. The interesting fact is that the resolved $\tilde{G}$ field does not have to follow the progress variable gradient. It can be smoothed out in space as broad as several mesh elements so that it may be resolved on the LES mesh. Simulation of $\tilde{G}$ field requires the solution of a transport equation where the displacement speed is modelled using the turbulent flame speed [13]. The major difficulty is that the turbulent flame speed is yet not a welldefined quantity and no universal model formulation can be found. Several geometrical assumptions also have to be imposed to maintain the adequate simplicity of the model while artificial diffusivity has to be added to avoid numerical difficulties arising due to the formation of flame cusps [14, 15]. Therefore the successful integration of the level set formulation with the turbulent flow field also requires considerable research.

The thicken flame model [16], artificially enhances the flame front thickness so that it can be sufficiently resolved in the computational mesh. Thickening of the flame front could be achieved by multiplying the thermal diffusivity by a user defined factor $F$, as the flame thickness $\delta_{L}$ is given by the ratio between the thermal diffusivity and the laminar flame speed. However, it is required to maintain the true laminar flame propagation speed so that a correction for the reaction rate is required. Simple laminar flame theories suggest that the laminar flame speed is proportional to the product of thermal diffusivity and the pre-exponential factor of the Arrhenius reaction rate equation. Hence, correct reaction rate is achieved by dividing the pre-exponential factor by the same factor $F$. The major advantage of this formulation is that it can be used to study various phenomena such as ignition and wall flame interaction, without the use of ad-hoc sub models as the reaction rate is modelled using the Arrhenius law [17]. Applications of this model for gas turbine combustion analysis $[18,19]$ and SI engines [20] have been reported. Extension of this study to analyse cyclic variations of combustion instabilities in SI engine combustion has also been reported in [21]. However, since the use of this method is combined with Arrhenius type reaction model, the flame region has to be accurately resolved for a reasonable solution. On the other hand, the thicken flame model requires the use of a combustion filter width, which is at least larger than 10 times the cell size (the classical LES filter width). For better results this can be up to fifty times [17]. Hence, compared to the other methods, thicken flame approach is more demanding in terms of the mesh resolution requirement [22].

Another interesting approach to solve the flame thickness problem is to filter the progress variable (or species mass fraction) by a larger filter size than the classical LES filter width [23]. The present study is based on this technique and uses the flame surface density (FSD) model as further explained in the following sections. In this work, attempts have also been made to develop an LES based dynamic procedure for turbulent premixed combustion calculations in Page 2 of 14 spark ignited gasoline engines. The work is based on the wellknown KIVA-4 code. It is the latest of RANS based CFD flow solvers of KIVA series, capable of simulating threedimensional, multispecies gaseous flows under steady and transient conditions. KIVA solves the governing equations in an Arbitrary Lagrangian Eularian framework and has the ability to handle unstructured meshes with moving boundaries. In this study, the code was extensively modified to incorporate LES capability by implementing a sub-grid scale turbulence model, where a separate transport equation is solved for the sub-grid kinetic energy. An improved model for the spark ignition and flame kernel formation in the LES context was also developed. Fully developed phase of the combustion was simulated using a dynamic flame surface density approach. Full cycle simulations were carried out for a number of engine cycles and predictions were compared against experimentally measured data for the same engine.

\section{NUMERICAL PROCEDURE}

Described in this section are the Favre filtered governing equations solved during the present study for a premixed reacting flow field. A comprehensive discussion on the derivation of these equations can be found in [24, 25]. Specific aspects of implementation into the KIVA-4 code is explained in [26]. The momentum equation applied in the $x$ direction for example, can be written as:

$$
\begin{aligned}
\frac{\partial}{\partial t}(\bar{\rho} \tilde{u})+\nabla \cdot(\bar{\rho} \tilde{u} \widetilde{U}) & +\nabla \cdot \tau_{s g s_{-} x} \\
= & -\frac{\partial \bar{p}}{\partial x}+\frac{\partial \bar{\tau}_{x x}}{\partial x}+\frac{\partial \bar{\tau}_{y x}}{\partial y}+\frac{\partial \bar{\tau}_{z x}}{\partial z}
\end{aligned}
$$

where, $U$ is the velocity vector and $u$ is its component in $x$ direction. $\rho$ is the fluid density and $p$ is the pressure. The SGS tensor is denoted by $\tau_{s g s}$ and $\tau_{s g s_{-} x}$ corresponds to its components in $x$ direction. $\tau_{y x}$ indicates the stress component acting in $y$ plane in $x$ direction. The resolved stress tensor $\tau$ can be obtained by filtering the Newtonian stress tensor as:

$$
\tau=\mu\left[(\nabla \otimes \widetilde{U})+(\nabla \otimes \widetilde{U})^{\mathbb{T}}\right]-\frac{2}{3} \mu(\nabla \cdot \widetilde{U}) \mathbb{I}
$$

where, $\mathbb{I}$ is the unit tensor and $\mathbb{T}$ is the transpose. $\mu$ represents the dynamic viscosity and $\otimes$ denotes the tensor product. The closure of the momentum equation requires an expression for the SGS stress tensor. In this study, an approach based on the SGS kinetic energy: $k_{s g s}[24]$, is taken.

The assembled SGS stress tensor can be given by:

$$
\begin{aligned}
\tau_{s g s}=-\mu_{s g s}\left[(\nabla \otimes \widetilde{U})+(\nabla \otimes \widetilde{U})^{\mathbb{T}}\right] \\
+\frac{2}{3}\left[(\nabla \cdot \widetilde{U})+\bar{\rho} k_{s g s}\right] \mathbb{I}
\end{aligned}
$$


The SGS viscosity: $\mu_{s g s}$, is evaluated by the following expression.

$$
\mu_{s g s}=C_{v} \bar{\rho} k_{s g s}^{1 / 2} \bar{\Delta}
$$

$C_{v}$ is a model constant usually taken to be 0.067 for engine configurations [24,27]. SGS kinetic energy is obtained by solving the following transport equation.

$$
\begin{aligned}
\frac{\partial}{\partial t}\left(\bar{\rho} k_{s g s}\right)+\nabla \cdot & \left(\bar{\rho} k_{s g s} \widetilde{U}\right) \\
& =-(\nabla \otimes \widetilde{U}): \bar{\tau}_{s g s}-D_{s g s} \\
& +\nabla \cdot\left(\frac{\mu_{s g s}}{S c_{k}} \nabla k_{s g s}\right)
\end{aligned}
$$

Balance equations for the species and global mass conservation can be specified as:

$$
\begin{gathered}
\frac{\partial \bar{\rho}}{\partial t}+\nabla \cdot(\bar{\rho} \widetilde{U})=0 \\
\frac{\partial\left(\bar{\rho} \widetilde{Y}_{m}\right)}{\partial t}+\nabla \cdot\left(\bar{\rho} \widetilde{Y}_{m} \widetilde{U}\right)-\nabla \cdot\left(\frac{\mu}{S c} \nabla \tilde{Y}_{m}\right)+\nabla \cdot \Phi_{s g s} \\
=\bar{\rho}_{m, c} \\
\Phi_{s g s}=-\left(\frac{\mu_{s g s}}{S c_{s g s}} \nabla \tilde{Y}_{m}\right)
\end{gathered}
$$

where, $Y_{m}$ is the mass fraction of species $m$. The unresolved convective fluxes are denoted by $\Phi_{s g s}$ and closed using the classical gradient assumption. $\bar{\rho}_{m, c}$ denotes the source term due to combustion. Similarly, the transport of specific internal energy $I$ can be written as:

$$
\begin{gathered}
\frac{\partial}{\partial t}(\bar{\rho} \tilde{I})+\nabla \cdot \bar{\rho} \tilde{I} \widetilde{U}+\bar{p}(\nabla \cdot \widetilde{U})+\nabla \cdot \bar{q}-(\nabla \otimes \widetilde{U})^{\mathbb{T}}: \bar{\tau} \\
+\nabla \cdot h_{s g s}-D_{s g s}=\bar{Q}_{I, c} \\
h_{s g s}=-\frac{\mu_{s g s} c_{p}}{P r_{s g s}} \nabla \cdot \tilde{T} \\
D_{s g s}=C_{\varepsilon} \bar{\rho}^{3 / 2}{ }^{3 / 2} / \bar{\Delta}
\end{gathered}
$$

where, $h$ is the enthalpy and $T$ is the temperature. $\bar{Q}_{I, c}$ is the combustion source term and $c_{p}$ is the specific heat capacity at constant pressure. $D_{s g s}$ represents the rate of dissipation of SGS kinetic energy.

The heat flux vector $q$ is given by:

$$
\bar{q}=-\mathcal{K}(\nabla \tilde{T})-\sum_{m} \frac{\mu}{S c} \tilde{h}_{m}\left(\nabla \tilde{Y}_{m}\right)
$$

$\mathcal{K}$ is the thermal conductivity of the fluid mixture and the summation is over all the species.

The LES filter width is taken as:

$$
\bar{\Delta}=(\Delta x \Delta y \Delta z)^{1 / 3}
$$

where, $\Delta x, \Delta y$ and $\Delta z$ are length, width and height of the computational cell, thus the cut off scale is often taken equivalent to the cubic root of the cell volume. Rest of the modelling constants were assigned the following values following [24]. $C_{\varepsilon}=0.916 S c_{k}=1.0 P r_{s g s}=0.9 S c_{s g s}=$ 0.9 . The implementation of this model in the KIVA-4 code has been successfully validated with respect to experimental and DNS data as reported in [26].

\section{Modelling the Reaction Rate Using a FSD Approach}

It is also possible to extend the FSD concept; i.e $\Sigma$ : the available flame surface area for unit volume, developed in RANS to perform combustion calculations in LES, in conjunction with the filtered balance equation of progress variable or species mass fractions [10]. However, as already pointed out, the stiffness associated with the spatial distribution in progress variable has to be eliminated in order this method to be successful.

\section{Combustion Filter Width}

It has been demonstrated that if the flame front is filtered with a larger filter size $\bar{\Delta}_{c}$ than the classical LES filter width $\bar{\Delta}$, a similar effect can be seen as the thicken flame model [23]. For example, if the combustion filter width $\bar{\Delta}_{c}$ is taken to be 4 times the grid filter width $\bar{\Delta}$, the progress variable gradient is dispersed over $2 \bar{\Delta}_{c} / \bar{\Delta}$ grid points $\left(2 \bar{\Delta}_{c} / \bar{\Delta}=8\right.$ in this case). In practice, the flame front is thickened by a factor in the order of $\bar{\Delta}_{c} / \delta_{L}$ [22] making it possible to resolve with a sufficiently large filter width.

The resolution parameter $\eta_{\text {res }}$ is defined by relating combustion and grid filter widths as:

$$
\bar{\Delta}_{c}=\eta_{\text {res }} \bar{\Delta}
$$

It has also been highlighted in [23] that there should be at least eight grid points for the accurate solution of the flame front. Therefore in practice $\eta_{\text {res }}$ is chosen such that $5<\eta_{\text {res }}<10$ [9].

It should be noted here that the filter width $\bar{\Delta}_{c}$ is applied only to the flame region and for the rest of the domain the classical 
filter width $\bar{\Delta}$ must be used. In addition, $\bar{\Delta}_{c}$ is used only with the species (or progress variable) and enthalpy transport equations. In the momentum equations, $\bar{\Delta}$ is used all over the domain including the flame front region. In a practical CFD simulation it is difficult to exactly identify a flame region, therefore the combustion filter width may be used only in the region where filtered progress variable $10^{-3} \leq \tilde{c} \leq 1-10^{-3}$ as recommended in [9].

Use of the filter width in governing equations is associated with the calculation of the SGS viscosity. Hence, the SGS viscosity in the flame region $\mu_{s g s-c}$, has to be calculated as follows.

$$
\mu_{s g s-c}=C_{v} \bar{\rho} k_{s g s-c}^{1 / 2} \bar{\Delta}_{c}
$$

where, $k_{s g s-c}$ is taken as:

$$
k_{s g s-c}=k_{s g s}\left(\frac{\bar{\Delta}_{c}}{\bar{\Delta}}\right)^{2 / 3}
$$

assuming Kolmogorov energy cascade in the inertial sub range. Consequently, the balance equation for mass fraction, enthalpy or progress variable have to be modified to incorporate combustion filter scale viscosity as given in the form below for a general scalar $\phi$.

$$
\frac{\partial \bar{\rho} \tilde{\phi}}{\partial t}+\nabla \cdot(\bar{\rho} \tilde{\phi} \widetilde{U})-\nabla \cdot\left[\left(\frac{\mu}{S c}+\frac{\mu_{s g s-c}}{S c_{s g s}}\right) \nabla \tilde{\phi}\right]=S_{\phi}\left(\bar{\Delta}_{c}\right)
$$

Also, it is worth mentioning that the combustion source term in the above equation is a function of the combustion filter width $\bar{\Delta}_{c}$.

\section{Transport of the Progress Variable}

The modified equation suggested in [28] for the Favre filtered progress variable: $\widetilde{c}$ is adopted in the present study to solve for the species mass fraction $\tilde{Y}_{m}$.

$$
\begin{aligned}
& \frac{\partial\left(\bar{\rho} \widetilde{Y}_{m}\right)}{\partial t}+\nabla \cdot\left(\bar{\rho} \widetilde{Y}_{m} \widetilde{U}\right) \\
&+\nabla \cdot\left[\left(\rho_{u} \frac{S_{L} \bar{\Delta}_{c}}{16 \sqrt{6 / \pi}}+\frac{\mu_{s g s-c}}{S c_{s g s}}\right) \nabla \tilde{Y}_{m}\right] \\
&=\left\langle\rho_{u} \tilde{Y}_{m} S_{D}\right\rangle_{s} \bar{\Sigma}
\end{aligned}
$$

where, $\bar{\Sigma}=\overline{|\nabla c|}$ denotes a generalized filtered flame surface density and, is a measure of total flame surface area contained within the filtered volume. The density weighted surface averaged displacement speed $\left\langle\rho S_{D}\right\rangle_{S}$ is generally approximated in terms of the unburned gas density $\rho_{u}$, and laminar flame speed $S_{L}$.

Page 4 of 14

$$
\left\langle\rho S_{D}\right\rangle_{S}=\rho_{u} S_{L}
$$

$\bar{\Sigma}$ can be evaluated by solving a balance equation for the transport of flame surface density, making necessary allowance for the unresolved contribution [9,29]. However, these models often involve several model constants which need to be specified on case by case basis. Alternatively, a simpler but an attractive approach is to use an algebraic model formulation which may only have one or two calibration constants. The algebraic model developed here, virtually involve no adjustable contestant, as they are dynamically estimated using the resolved scale information. In fact, the potential of dynamic algebraic formulations in predicting reacting SI engine flows have never been tested. Thus, with the aim of gaining confidence in premixed turbulent combustion in SI engines the, the present work adopts an algebraic FSD formulation.

\section{A Flame Kernel Growth Model}

Combustion characteristics during the early stage of kernel formation, is fundamentally different from its fully developed phase $[8,30]$. Flame has not achieved equilibrium and flame propagation is mainly affected by volumetric expansion. Instead, as the flame kernel remains smaller than the computational mesh during the early stage of propagation, the flame front cannot be resolve in the computational mesh [31]. Thus, combustion models, usually developed with equilibrium assumptions for fully developed flames are not valid for this stage and specific modifications are needed. Only a very limited amount of work has been carried out in LES context, on ignition and flame kernel formation in SI engines. In [31] and [32], FSD transport equation based ignition models have been developed considering aforementioned aspects. Recently, an experimentally based comprehensive formulation combined with the G-equation approach has also been proposed in [33] and [34] for RANS simulations. This would, probably be easily extended in to LES, in a LES G-equation formulation. However, in the present work, a simple model, but effectively capable of producing global results of ignition and early stage of flame propagation is proposed.

\section{Initiation of the Ignition Kernel}

The most accurate method to initiate the ignition is to impose a plasma channel, based on the characteristics of the electrical system and in-cylinder mixture properties. Solving for the complex plasma physics is usually a formidable task. Hence, in the present approach the flame kernel is initiated by depositing a burned gas mass of $m_{i g n}$ in the spark location. Breakdown and arc discharge period is simply neglected as the total duration of these events is only few micro seconds. If necessary data from the ignition system is available, $m_{i g n}$ can directly be estimated from the relations given in [35]. A simplified approach is adopted in the present study as no data was available on the ignition system. Onset of the kernel is assumed to be spherical with a diameter of $d_{e}$ : the spark 
electrode gap, and the burned gas at adiabatic temperature. Consequently, $m_{\text {ign }}$ can be calculated from the below expression:

$$
m_{i g n}=\frac{\pi}{6} \rho_{u} d_{e}^{3}
$$

This burned gas volume is filtered using the combustion filter width $\bar{\Delta}_{c}$ by adopting the following Gaussian relation [32].

$$
\tilde{c}=c_{0} \exp \left[\frac{-\left|x-x_{s p k}\right|^{2}}{0.6^{2} \bar{\Delta}_{c}^{2}}\right]
$$

$x-x_{s p k}$ is distance measured from the spark location. The constant $c_{0}$ is estimated such that

$$
\int \bar{\rho} \tilde{c} d V=m_{i g n}
$$

\section{Mean Flame Kernel Surface Area Evolution}

During the early stage of flame development, the flame kernel size is smaller than the combustion filter width so that the flame details are naturally filtered out. As a result $\bar{\Sigma}$ has to be estimated with physical arguments. It is assumed that the flame kernel is roughly spherical so that the volume of the flame kernel can be taken approximately equal to the total volume occupied by burned gas $V_{k}$. Consequently, the mean flame kernel radius $r_{k}$ and the flame area $A_{k}$ corresponding to the burned gas volume are defined respectively as [9]:

$$
\begin{gathered}
r_{k}=\left(\frac{3 V_{k}}{4 \pi}\right)^{1 / 3} \\
A_{k}=4 \pi r_{k}^{2}
\end{gathered}
$$

The flame wrinkling factor $\Xi$ is now defined as the ratio between the total flame surface area $A$ and the surface area of the burned gas volume $A_{k}$. Accordingly, $\Xi$ and flame surface densities can also be related by:

$$
\Xi=\frac{A}{A_{k}}=\frac{\bar{\Sigma}}{\bar{\Sigma}_{k}}
$$

where, $\bar{\Sigma}_{k}$ is the FSD corresponding to the burned gas volume and the reaction rate expression is now modified as:

$$
\overline{\dot{\rho}}_{c, u}=\rho_{u} \Xi \bar{\Sigma}_{k} S_{D}
$$

A relation for the flame area evolution of a thin flame kernel can be formulated as [36]:

$$
\frac{1}{A} \frac{d A}{d t}=\frac{1}{A_{k}} \frac{d A_{k}}{d t}+K_{W}
$$

However, under practical situations with finite flame thicknesses, this relation largely underpredicts the kernel growth rate [37]. As a solution the following modified expression is suggested [37].

$$
\frac{d A}{d t}=8 \pi r_{k} \frac{\rho_{u}}{\rho_{b}} S_{D}\left(\frac{A}{A_{k}}\right)^{\Psi}+K_{W} A
$$

The two terms in the right hand sides correspond to the strain rate due to volumetric expansion and turbulent flame wrinkling. Volumetric strain may be neglected for fully developed flames, but has to be estimated properly in flame kernel modelling as it is in the same order or even higher than the turbulent strain. The exponent $\Psi$ was approximated to be close to 2.0 based on DNS results [37] or it can be verified by simple theoretical analysis.

\section{Local Flame Surface Density}

The local mean flame surface density is calculated assuming a parabolic profile similar to the expression given in [28]. Note that $\xi$ may be different from the original values proposed in [28] as the flame propagation has not yet reached the turbulent phase. Nevertheless, $\xi$ was assumed to be a global time varying coefficient independent of the filter width.

$$
\bar{\Sigma}=\xi \frac{\tilde{c}(1-\tilde{c})}{\bar{\Delta}_{c}}
$$

Local mean flame surface area within a cell: $A_{c}$, is then approximated by integrating over the cell volume $v_{c}$.

$$
A_{c}=\xi \int_{v_{c}} \frac{\tilde{c}(1-\tilde{c})}{\bar{\Delta}_{c}} d V
$$

The total mean flame kernel area $A_{k}$, can then be calculated by integrating the local values in the burned gas volume.

$$
A_{k}=\xi \int_{V_{k}} \frac{\tilde{c}(1-\tilde{c})}{\bar{\Delta}_{c}} d V
$$

If the filter width is assumed to be uniform in space, local mean flame surface area can be related to the flame area calculated using the burned gas surface area [31].

$$
A_{c}=A_{k} \frac{\int_{v_{c}} \tilde{c}(1-\tilde{c}) d V}{\int_{V_{b}} \tilde{c}(1-\tilde{c}) d V}
$$

It is worth to note that the use of this procedure has eliminated the parameter $\xi$ from the final expression. 


\section{SGS Flame Wrinkling Factor}

The term $K_{W}$ in Eq. (31) is modelled as:

$$
K_{W}=\Gamma \frac{u_{\Delta_{e}}^{\prime}}{\bar{\Delta}_{e}}\left\langle\frac{\Xi_{E Q}-\Xi}{\Xi_{E Q}-\Xi_{t=0}}\right\rangle
$$

$\Gamma$ is the LES version of the ITNFS stretch function, evaluated at filter scale $\bar{\Delta}_{e}$. As the flame at early stage has not reached equilibrium, a linear evolution of $K_{W}$ is assumed. $K_{W}$ vanishes, when the flame reaches equilibrium. $\Xi$ is taken equal to the area ratio in equation 25. Angle brackets denote the flame averaging operation. The initial value of the wrinkling factor is taken as $\Xi_{t=0}=1$. The equilibrium wrinkling factor $\Xi_{E Q}$ can be expressed as follows [38].

$$
\Xi_{E Q}=\left[1+\min \left(\frac{\bar{\Delta}_{e}}{\delta_{L}}, \Gamma \frac{u_{\Delta_{e}}^{\prime}}{S_{L}}\right)\right]^{0.5}
$$

where, the laminar flame thickness here corresponds to the thermal flame thickness: $98 \%$ of the reaction zone width, and may be obtained using the fact that flame Reynolds number $R e_{f} \approx 4$ [39].

$$
R e_{f}=\frac{\delta_{L} S_{L}}{v} \approx 4
$$

The ITNFS function calculates the total stretch induced on the flame surface by all the eddies smaller than a given characteristic cut off size $\bar{\Delta}_{e}$ [38]. During the early stage of flame kernel formation, flame dimension is smaller than the size of the integral scale and the combustion filter width. Hence, the largest possible eddy size, which can wrinkle the flame, is in the order of the flame kernel diameter: $d_{k}$. Consequently, the use of combustion filter width as the characteristic cut off width over estimates the winkling factor. Hence, $\bar{\Delta}_{e}$ has to be replaced by the flame kernel diameter as long as the flame dimension remains smaller than the combustion filter width. Hence, the characteristic cut off width is defined as:

$$
\bar{\Delta}_{e}=\min \left(d_{k}, \bar{\Delta}_{c}\right)
$$

Accordingly, $u_{\Delta_{e}}^{\prime}$ now corresponds to the velocity scale of eddies of the flame dimension. Assuming that SGS scales are in the inertial sub range following Kolmogorov energy cascade, the SGS velocity in the grid scale $(\bar{\Delta})$ and the characteristic scale can be related by:

$$
u_{\Delta_{e}}^{\prime}=\left[\frac{\min \left(d_{k}, \bar{\Delta}_{c}\right)}{\bar{\Delta}}\right]^{1 / 3} u_{\Delta}^{\prime}
$$

\section{Plasma Expansion Speed}

At the initial stage, the flame propagation speed is enhanced by the effect of the plasma expansion. Hence, the effective displacement speed is the summation of laminar and plasma speeds [40].

$$
S_{D}=S_{\text {plasma }}+S_{L}
$$

$S_{\text {plasma }}$ is the mean expansion speed of the high temperature plasma kernel during the glow discharge phase. The following expression is adopted from [41] by suitably modifying with LES filtered quantities.

$$
S_{\text {plasma }}=\frac{\eta \dot{Q}_{\text {spark }}}{A_{k}\left[\bar{\rho}_{u}\left(\widetilde{U}_{k}-\tilde{h}_{u}\right)+p_{k} \frac{\bar{\rho}_{u}}{\bar{\rho}_{k}}\right]}
$$

$U$ and $h$ are respectively the specific internal energy of and enthalpy. $\dot{Q}_{\text {spark }}$ is the rate of spark energy deposition. The efficiency factor $\eta$ is taken to be 0.2 [9]. The burned gas pressure $p_{k}$ is assumed to be uniform within the kernel.

\section{Transition to the Main Combustion Model}

Flame surface density approach assumes that there exists fully burned gas behind the flame front. Therefore ignition model is employed until a fully resolvable flame front is developed so that progress variable becomes unity somewhere in the combustion domain. Beyond this point, the combustion rate is calculated by the main combustion model. This approach has been successfully used in $[9,31]$.

\section{A Dynamic FSD Model for the Fully Developed Phase of Combustion}

In LES, the resolved part of the FSD can be computed from the resolved flow properties and the SGS part has to be modelled. Boger et al. [23] was the first to come up with an algebraic expression for $\bar{\Sigma}$ after extensive analysis of DNS data of infinitely thin flames. The analysis is based on filtering the balance equation of progress variable with a Gaussian filter having a cut off width $\bar{\Delta}_{c}$ large than the mesh size $\bar{\Delta}$ to ensure the filtered flame front is numerically resolved in the computational mesh. The deduced expression is of the parabolic form given by:

$$
\bar{\Sigma}=4 \beta \Xi \frac{\tilde{c}(1-\tilde{c})}{\bar{\Delta}_{c}}
$$

where, $\beta$ is a model constant, estimated to be $\sqrt{6 / \pi}$ via analytical derivation [23]. However, the above formulation only provides an approximation for the resolved FSD. The factor $\Xi$ accounts for the SGS flame wrinkling effects. $\Xi$ may be used as a tuning constant or modelled using an algebraic 
expression or a balance equation. Applications of this model in modelling early stage flame development has been reported in [22] and in coupled radiative heat transfer calculations in [42]. Another promising approach is to solve a transport equation for the wrinkling factor as in [43]. Alternatively, the SGS contribution of FSD can be explicitly calculated from the resolved quantities [44-47].

The approach used here evaluates $\Xi$ using a dynamic procedure. Following [22], $\Xi$ is modelled as the ratio between the combustion filter scale and the inner cut off scale. $\bar{\Delta}_{c}$ and $\delta_{c}$ are respectively the largest and the smallest possible flame wrinkling scales.

$$
\Xi\left(\bar{\Delta}_{c}\right)=\left(\frac{\bar{\Delta}_{c}}{\delta_{c}}\right)^{\alpha}
$$

In the case of a fully fractal flame $\alpha$ may reduce to $D-2$; where $D$ is the fractal dimension. However, $\alpha$ is herein considered as an exponential factor in general.

A Germano like identity for the flame surface density can be formulated as:

$$
\hat{\bar{\Sigma}}=\hat{\Sigma}
$$

$\hat{\Sigma}$ is the FSD evaluated at test filter scale $\hat{\Delta}$ and $\hat{\bar{\Sigma}}$ is the FSD obtained by test filtering $\bar{\Sigma}$. If the flame surface density is modelled using the Boger's [23] relation, the above relation becomes:

$$
\left[4 \beta\left(\frac{\bar{\Delta}_{c}}{\delta_{c}}\right)^{\alpha} \frac{\tilde{c}}{(1-\tilde{c})} \bar{\Delta}_{c}\right]=4 \beta\left(\frac{\hat{\Delta}}{\delta_{c}}\right)^{\alpha} \frac{\hat{\tilde{c}}(1-\hat{\tilde{c}})}{\hat{\Delta}}
$$

Assuming the inner cut off scale to be independent of the filter width, it can further be rearranged to

$$
\alpha=1+\log \frac{\langle\tilde{c}(\overline{1-\tilde{c}})\rangle}{\langle\hat{\tilde{c}}(1-\hat{\tilde{c}})\rangle} / \log \left(\widehat{\Delta} / \bar{\Delta}_{c}\right)
$$

It has been mentioned that the use of this expression in practise may result in incorrect estimation of the wrinkling factor for laminar or nearly laminar like flames as $\alpha$ does not reach to zero under such conditions [22]. However, the effect of this formulation has never been practically investigated. Thus, as a first step the above expression is retained here. The gradient based method suggested in [22] would also be studied as the next step. Current approach is particularly attractive because of less computational time involved whereas, it needs a significant time to evaluate gradients particularly in unstructured grids. The inner cut off scale is taken here as three times the thermal flame thickness following [22, 44-47].

\section{$\underline{\text { Test Filtering Procedure }}$}

In the present study, a Gaussian test filter is used. Test filter width is taken to be twice the combustion filter width and combustion filter width is taken as five times the cell size. Accordingly, $\widehat{\Delta}=\sqrt{5} \bar{\Delta}_{c}$. Thus, test filtering domain involves nearly a thousand of neighbour cells per each cell. Storage of this information requires a huge amount of memory, which is unaffordable at present stage. Similarly, real time calculation also needs a very large computational time. In the work of Wang et al. [22], a separate computer code has been run in parallel just for test filtering purposes. In the present study, neighbour cell data and Gaussian weights were pre-calculated and stored in two direct access binary files. Records were appropriately read from these files as required. However, use of this procedure required to maintain a fixed mesh in both the space and time where vertex locations do not move. To facilitate this constrain piston motion was simulated only by adding and removing of cell layers, but avoiding being compressed or expanded.

Test filtering near solid boundaries require special measures. It is assumed that there exists an imaginary mesh outside the boundary, which has a similar mesh density as the actual computational mesh. For a reasonably uniform mesh, average number of test filtering cells per each cell and the summation of the Gaussian weights should also be uniform. Thus, averaged values of the Gaussian weights was used when test filtering the cells close to the boundary. A zero value for the progress variable is assumed in the imaginary mesh, similar to the zero padding technique in image processing [48].

\section{Testing and Validation Configurations}

Validation of the ignition and flame kernel model was performed by simulating the flame growth in an engine swirl chamber [38]. Flow and operating conditions of the simulated cases are given in Table 1.

Table 1. Simulated conditions of the swirl combustion chamber

\begin{tabular}{lcc}
\multicolumn{3}{c}{ chamber } \\
\hline \hline Test case No: & 1 & 2 \\
Engine RPM & 300 & 500 \\
Fuel & Propane & Propane \\
F/A equivalence ratio & $1.0 \&$ & $1.0 \& 0.769$ \\
Pressure at ignition (bar) & 0.769 & \\
Temperature at ignition(K) & 5.0 & 5.0 \\
Turbulent intensity (cm/s) & 660 & 660 \\
Mean flow velocity at peripheral & 44.0 & 73.0 \\
spark location $(\mathrm{cm} / \mathrm{s})$ & 750 & 1240 \\
Ignition energy supplied (mJ) & 60 & 60 \\
Ignition duration (ms) & 1.2 & 1.1 \\
\hline \hline
\end{tabular}


The validation was extended by modelling the full cycle combustion process in a Ricardo E6 experimental engine at Loughborough University. Specifications of the engine are given in the Table 2 .

\begin{tabular}{ll} 
Table 2. Geometric details of Ricardo E6 engine \\
\hline \hline Bore $(\mathrm{cm})$ & 7.62 \\
Stroke $(\mathrm{cm})$ & 11.11 \\
Squish* $(\mathrm{cm})$ & 1.4428 \\
Connecting Rod Length $(\mathrm{cm})$ & 24.13 \\
Intake Valve Opening & 009 - BTDC \\
Intake Valve Closing & 217 - ATDC \\
Exhaust Valve Opening & 147 - ATDC \\
Exhaust Valve Closing & 010 - ATDC \\
Max. Intake Valve Lift $(\mathrm{cm})$ & 1.156 \\
Max. Exhaust Valve Lift $(\mathrm{cm})$ & 1.06 \\
Fuel & Gasoline \\
\hline \hline
\end{tabular}

Computational grids for both cases comprised of unstructured hexahedron cells. The engine mesh, in total contains 0.8 million cells and corresponds to a nominal cell dimension of $0.7 \mathrm{~mm}$ within the combustion chamber. Simulated engine conditions correspond to 0.98 equivalence ratio at $1800 \mathrm{rpm}$ fuelled with gasoline. Onset of the spark was set at $20^{\circ}$ before top dead centre (TDC).

Continuous simulation of multiple LES engine cycles requires a huge amount of computational time. Therefore, parallelized computer codes are often required [9, 31, 40]. As the KIVA-4 code used in this work is a serial code, simulations were limited to a number of separate individual cycles, and the effects of cycle-by-cycle variation were introduced by superimposing random fluctuations on the mean intake pressure. However, this approach does not exactly mimic the actual cycle-by-cycle variations of flow properties. Multicycle LES simulations of an SI engine have been reported in [7, 9], where the first engine cycle (at the start of the simulation) has been modelled by superimposing random fluctuations similar to the present approach. Investigation of these results in [7,9], show that, the current technique is capable of representing such cycle-by-cycle variations to a reasonable degree. However, for more accurate results, continuous multi-cycle simulations are recommended.

Simulations of the Ricardo engine was started at 20 BTDC on exhaust stroke. Initial properties and mass fractions were calculated using a thermodynamic analysis. Based on exhaust gas temperature measurements, in-cylinder and exhaust gas mixture temperatures of the Ricardo E6 engine were taken to be $750 \mathrm{~K}$ at the start of simulation. In-cylinder, fluid and turbulent properties were homogeneously initialized with superimposed random Gaussian fluctuations. Measured mean intake manifold pressure was set at the intake boundary with superimposed random fluctuations (maximum of 5\% from the mean value) following [2]. These fluctuations are the only source of external randomness introduced.
Single step fuel oxidisation is assumed and the laminar flame speed was calculated with the empirical relations proposed in [49].

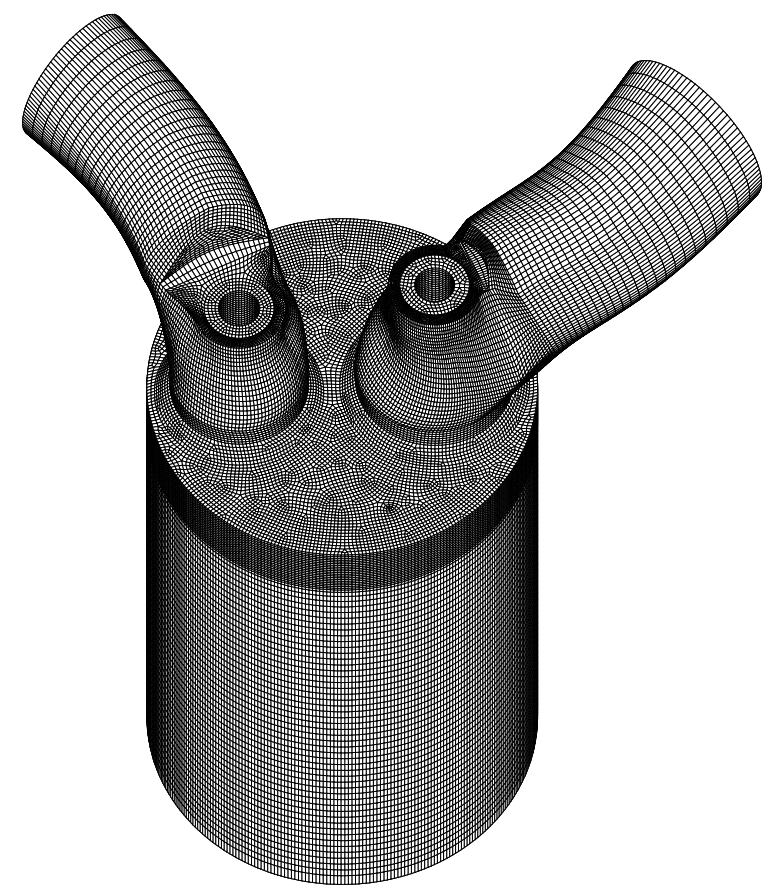

Figure 1. Computational mesh for the full cycle engine simulation of the Ricardo E6 engine

\section{RESULTS AND DISCUSSION}

\section{Validation of the Flame Kernel Model}

Shown in the figure 2 are the measured and predicted flame area evolutions for two simulated test cases in the swirl combustion chamber. The predicted area corresponds to the instantaneous surface area of the burned gas volume and the measured flame area has been calculated by averaging the flame area approximated using two dimensional schlieren photographs [30].

The predicted flame surface area is in close agreement with the measured flame area in all simulated test cases. A significant cyclic variation in flame area evolution has been observed in experiments [30]. However, the variations in the present simulations are very limited, due to the employed modelling strategy. Hence, only the results of a single simulation are presented for each of the operating condition. Basically, the simulation domain was taken as a closed chamber, whereas, in experiments it has been connected to the engine cylinder so that the flow properties inside are always dynamically changing. Simulation of the entire chamber requires a considerable simulation time and therefore, only the chamber volume is modelled. Initial swirl profile and the flow 
velocity were artificially initialized to match with experimentally measured mean values. Gaussian random fluctuations were superimposed to mean velocity field based on measured mean turbulent intensity. However, as often observed [50] this turbulent field decay very fast, so that effect of turbulence reduces with time.

\section{Case1}

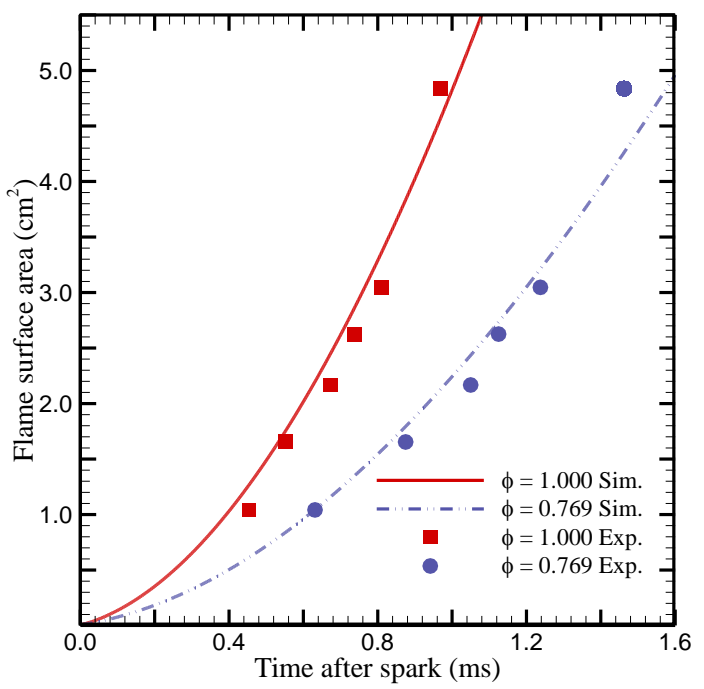

Case 2

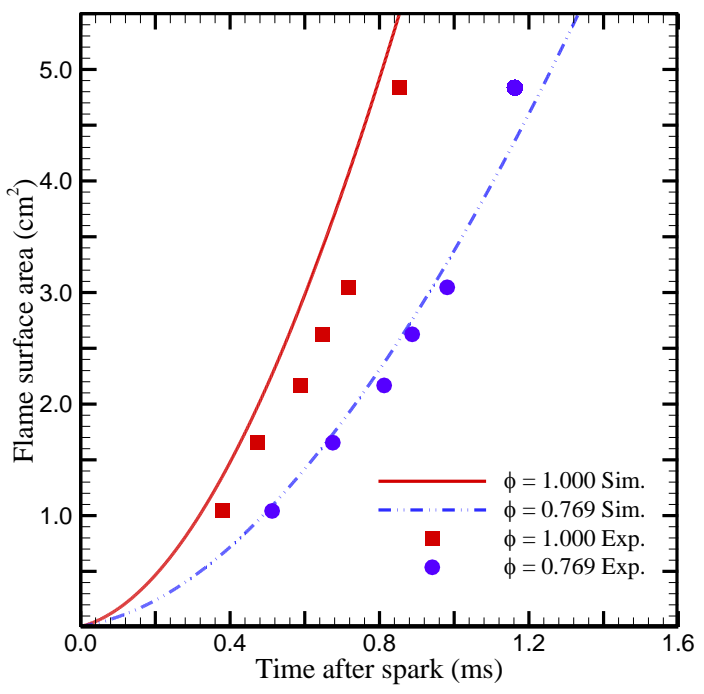

Figure 2. Comparison of measured and predicted flame kernel surface area for two different simulated test cases

During the simulations, a frozen turbulence field was assumed where the turbulent intensity and the laminar flame speed was kept constant. Thus, estimation of wrinkling factors are based the global values of these parameters. As a result, the predicted flame area remains very much close to the mean flame area without a significant variation. However, the model has been able to successfully predict the changes in the flame growth rates under different turbulent levels and fuel properties. Accordingly, this model should also be able to grasp localized effects of turbulent, particularly in engine combustion. This has been demonstrated in the figure 3 where the flame area growth from the ignition to the transition to the main combustion model is presented.

Only ten engine cycles were considered in the present study. It should be noted that ten cycles may not be sufficient to make a firm judgment of the cyclic variability of an engine. Hence, the results shown here are primarily used to demonstrate the predictability of the present formulation. Accordingly, the predicted flame area has shown significant variations even at the early stage of the flame propagation. Similarly, the variation of the transition point is seen to be considerable. The shortest time elapsed until the transition is about 4 crank degrees and the longest is close to 7 crank degrees. Importantly, it seems that this point is determined not just by the flame area growth rate, as no distinct relation can be identified between these two quantities.

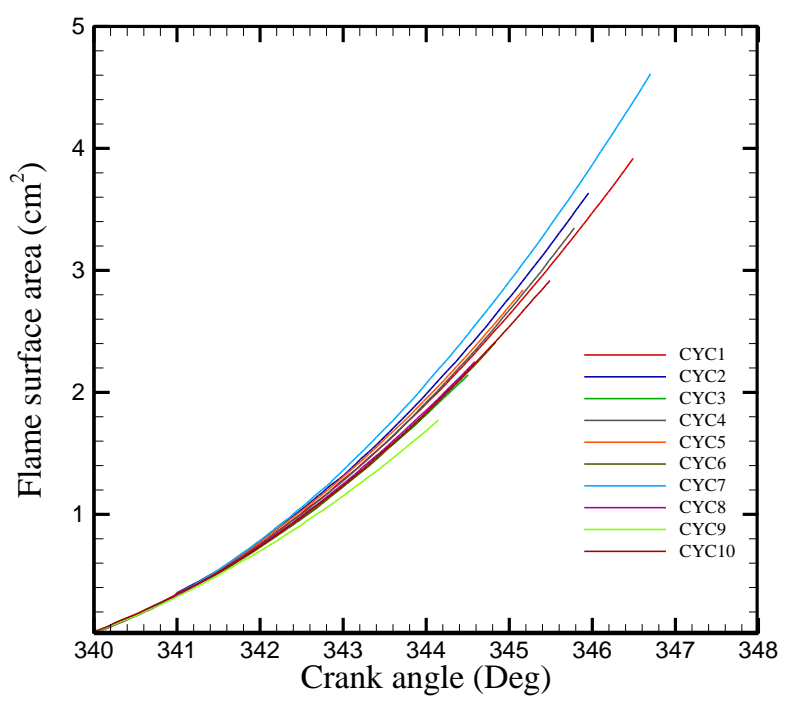

Figure 3. Evolution of the flame surface area during the early stage of flame kernel development in E6 engine. The end of each curve is the transition point

\section{Modelling Engine Combustion}

In-cylinder bulk flow motion is predominantly important in determining the overall combustion duration and macro flame structure. Spatial variations of the in-cylinder flow structures in three engine cycles at bottom dead centre during the intake stroke are shown in the figure 4. Results show that, even with relatively coarser meshes used in this work, present LES implementation has been able to resolve the evolution of a large number of in-cylinder flow structures. Particularly, flow features which are more influential for engine performance. This should be mainly due to the present SGS model where a separate transport equation is solved for SGS kinetic energy as pointed out in [51]. 

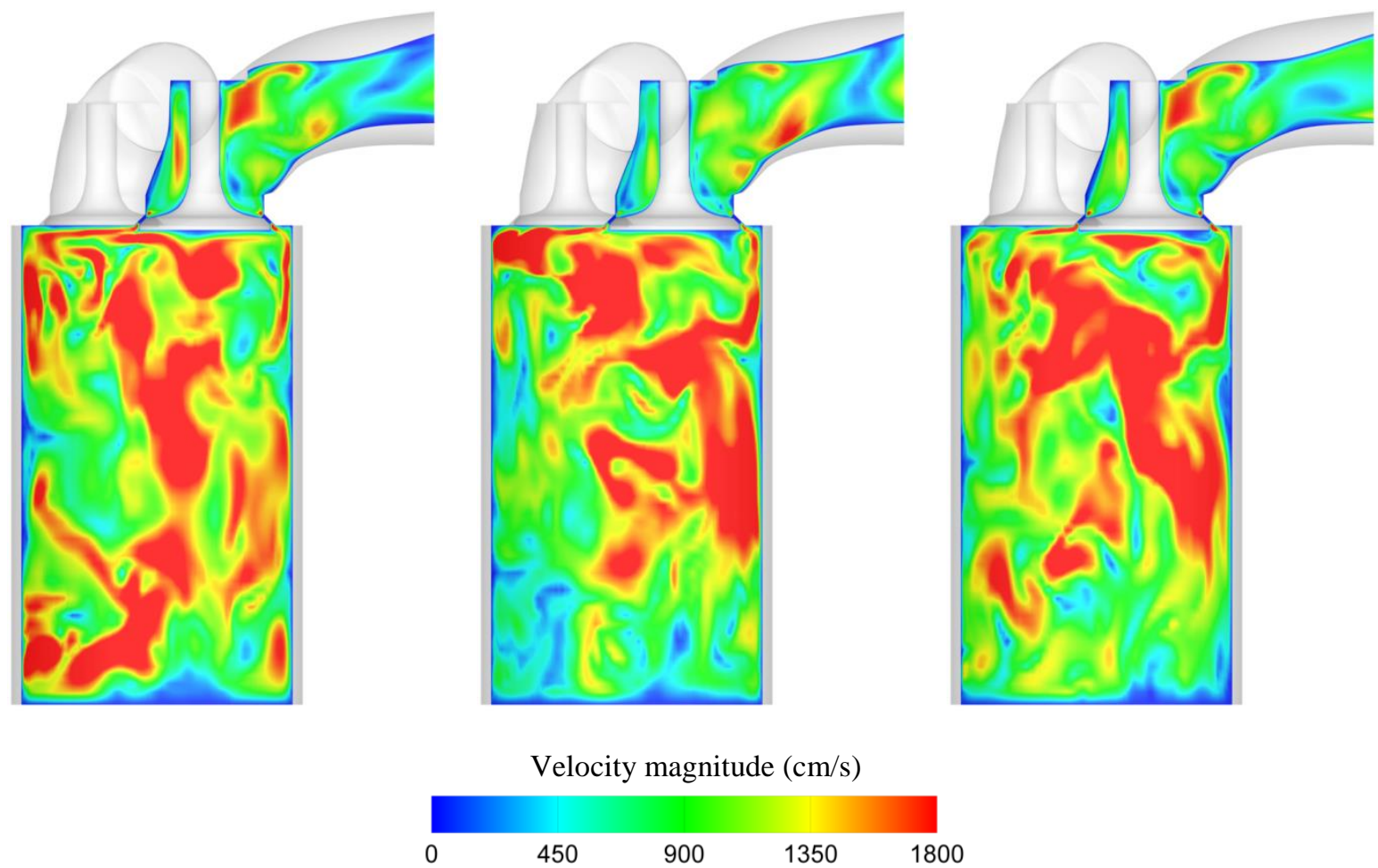

Figure 4. Variation of velocity magnitude across the intake valve plane at piston bottom dead centre during the intake stroke in three engine cycles
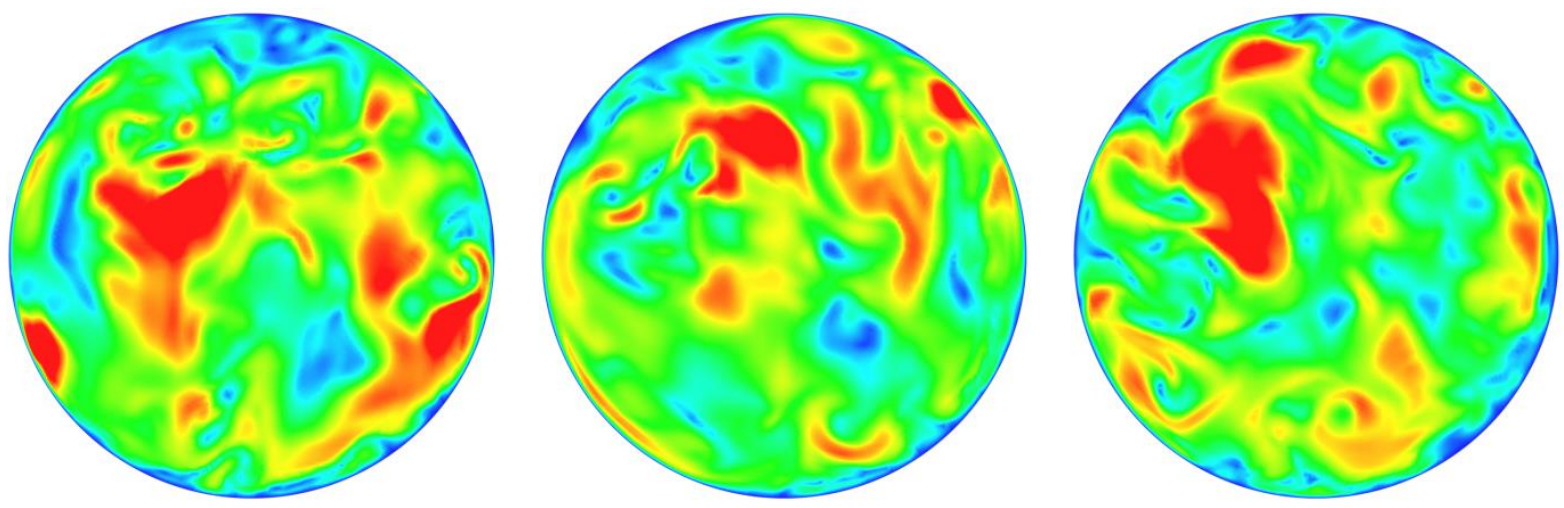

Velocity magnitude $(\mathrm{cm} / \mathrm{s})$

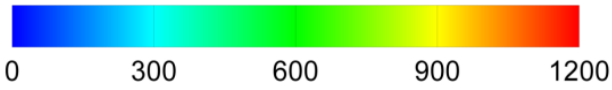

Figure 5. Variation of velocity magnitude across the spark plug plane just before ignition in three engine cycles

A rudimentary SGS model requires finer meshes for good results whereas, a well-engineered model would provide better results with coarser meshes. In addition, a significant cyclic variation of the bulk flow features are also apparent, which must effectively result in subsequent variations in the flame propagation. Similarly, in the figure 5, the variation of the velocity field close to the spark plug plane, prior to the ignition is shown. In all cases, the variations are found to be substantial in magnitude and such variations are very much expected in these types of single cylinder engines. As a result, the variations of the flame propagation and the pressure rise should also be noticeable.

Shown below in figure 6 are some of the most important combustion model parameters at two different crank angles. Illustrated in first row is the variation of filtered progress 
variable across the flame front while, second row shows the variation of test filtered progress variable. As expected, at test filter level wrinkling of the flame reduces but thickness increases. A smooth gradient has been maintained, near walls indicating that the current test filtering approach is capable of producing acceptable results. The local values of the wrinkling exponent $\alpha$ are shown in the third row. It was found to be close to zero at the leading edge of the flame front while, a value close to 1.0 in the fully burned region within the flame.

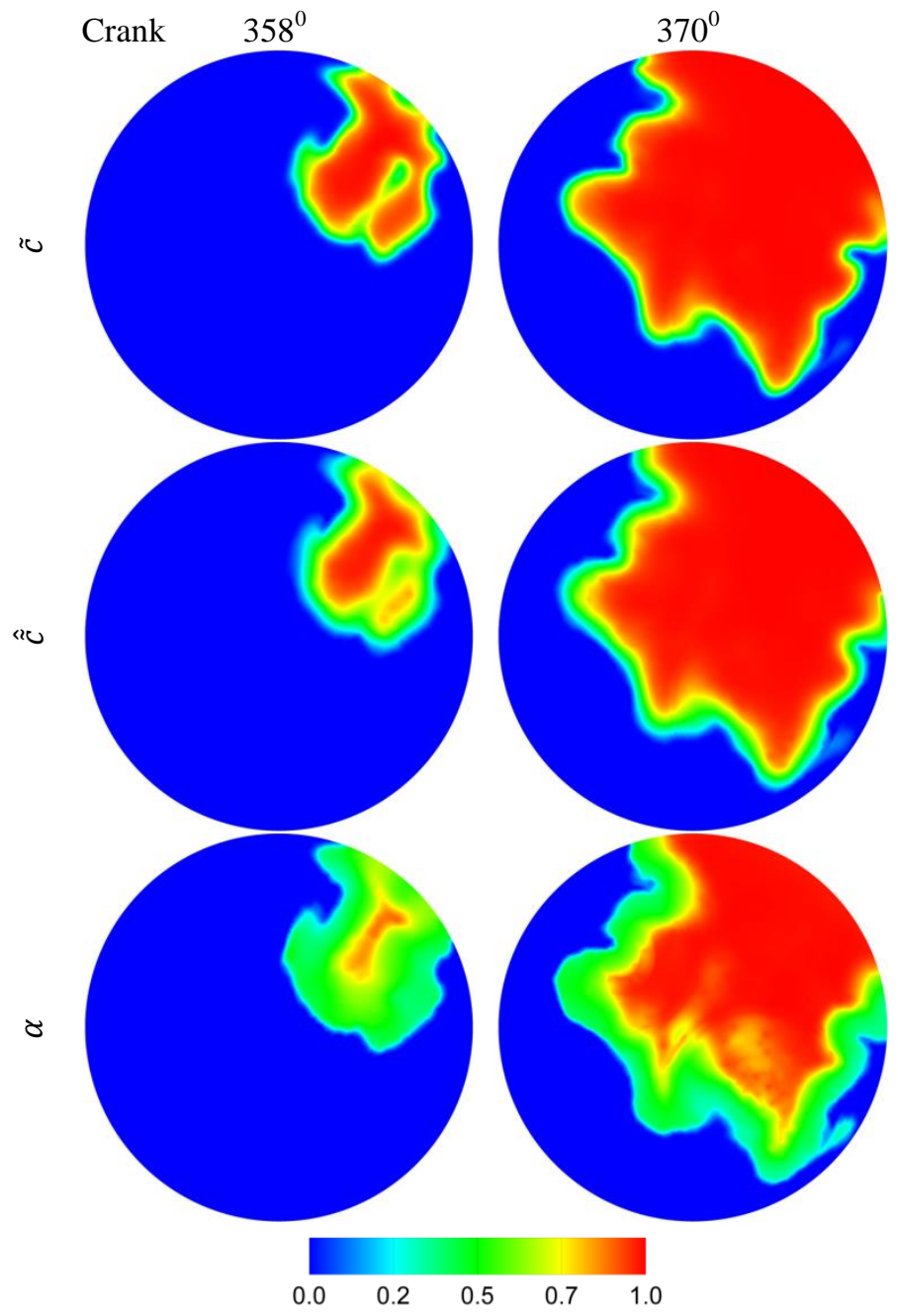

Figure 6. Distribution of the progress variable, test filtered progress variable and the exponent of the wrinkling term for two crank positions during the combustion phase

The reason for $\alpha$ to become unity is the presence of a near zero gradient of $\tilde{c}$ behind the reaction zone. Also, it has an average value of 0.5 inside the reaction zone. This is completely in agreement with the currently published literatures for these types of dynamic combustion models [22, 52]. This indicates a fractal dimension close to a value of 2.5. In general this value for engine applications is found to be quite high, compared to the typical range of $2.2-2.4$, often found in experimental literature [53].
Substantial variation in the instantaneous flame propagation is also observed for all the simulated engine cycles as presented in figure 7 . The flame front is represented by the iso- progress variable surface equal to 0.7 . Even though the instantaneous flame surfaces demonstrate significantly different localized geometric profiles, the global trend in the propagation direction and enflamed volume is shown to be the same. Wrinkling of the flame surface is clearly evidenced though out the flame propagation, more importantly the variations are apparent even from the very early stage of combustion.
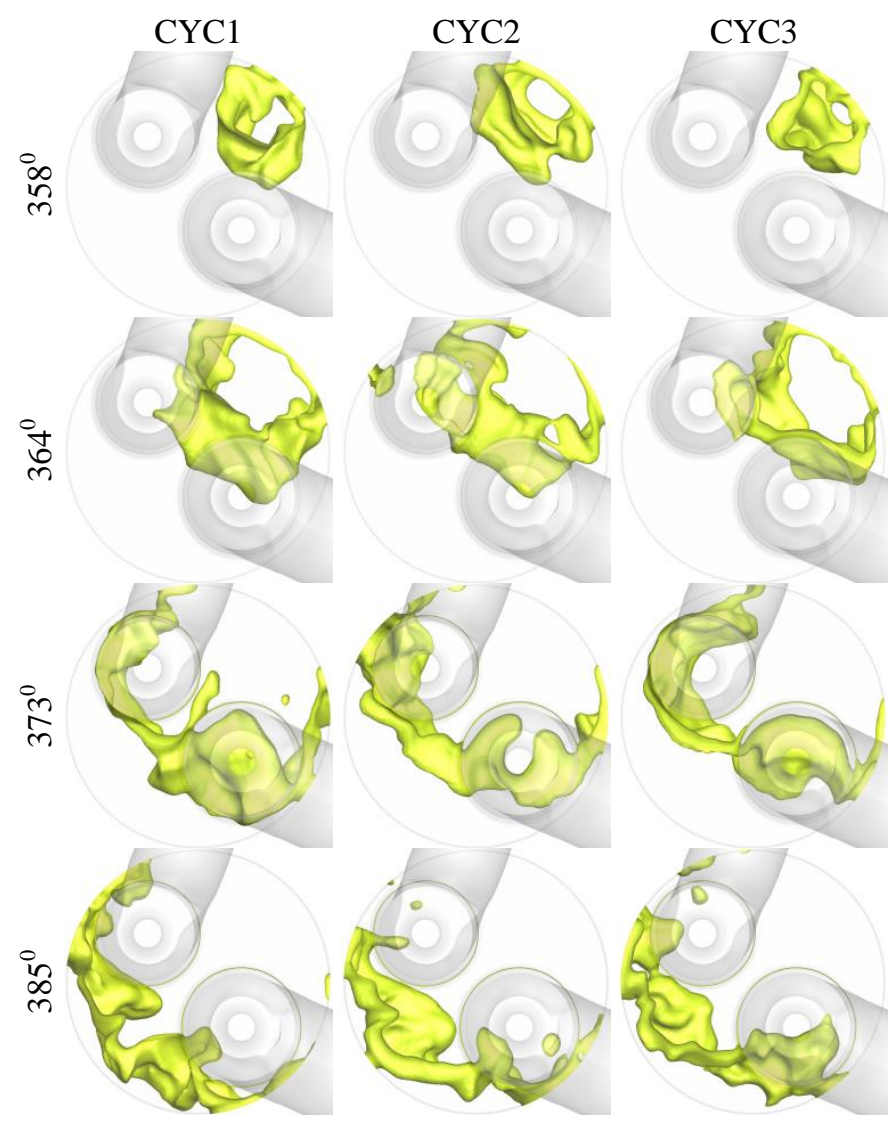

Figure 7. Flame surface propagation during three combustion cycles. Flame surface is represented by the isoprogress variable surface of $\tilde{c}=0.7$. Ignition at $340^{\circ}$

The main objective of an engine combustion model is to predict the in-cylinder pressure rise and the heat release rate during combustion. Present predictions of these parameters are shown in figure 8 and 9 respectively. The predicted pressure is well within the experimentally measured range and varies close to the cycle averaged pressure trace. The maximum variation of the experimental peak pressure from the cycle averaged value is slightly above $\pm 6 \mathrm{bar}$ and the simulated value is about \pm 3 bar. The difference, in the two quantities could mainly be a consequence of the present approach (imposing random fluctuations) used to specify the intake pressure boundary conditions for different engine cycles. Hence, it is clear that, initial assumption of the amplitude of 
pressure fluctuations (i.e. 5\% maximum) has caused a considerable under estimation of cycle-by-cycle variations.

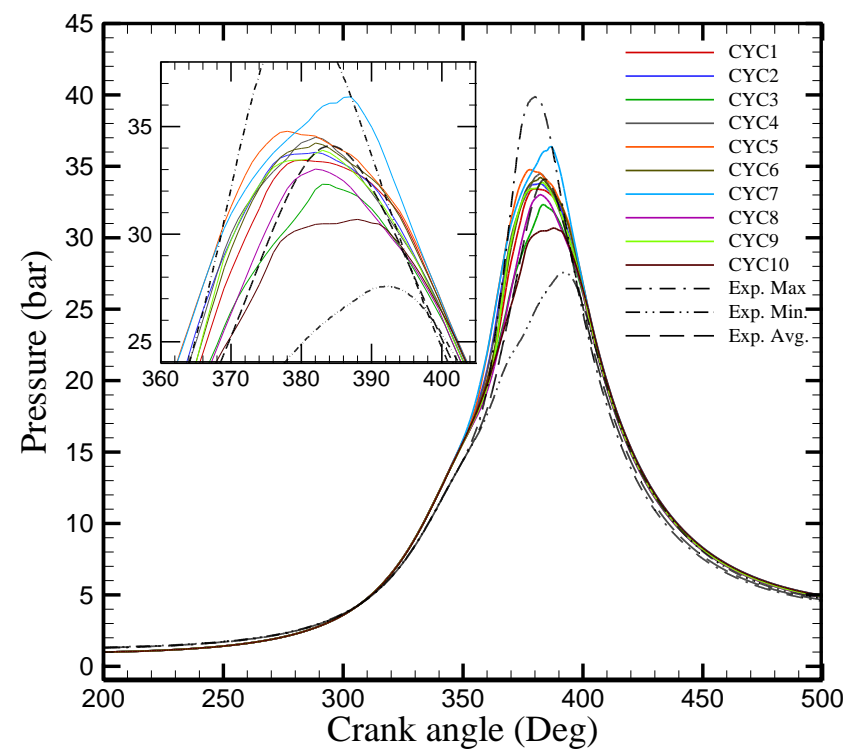

Figure 8. Comparison of computed and measured incylinder pressure variations for the Ricardo E6 engine

Note that, the magnitude of peak in-cylinder pressure during the cycle (figure 8) and the flame area growth rate during the early stage of the flame kernel formation (figure 3) are not directly interrelated for the present cases. This is caused by the differences in the trapped fuel mass and the variations in the in-cylinder turbulence.

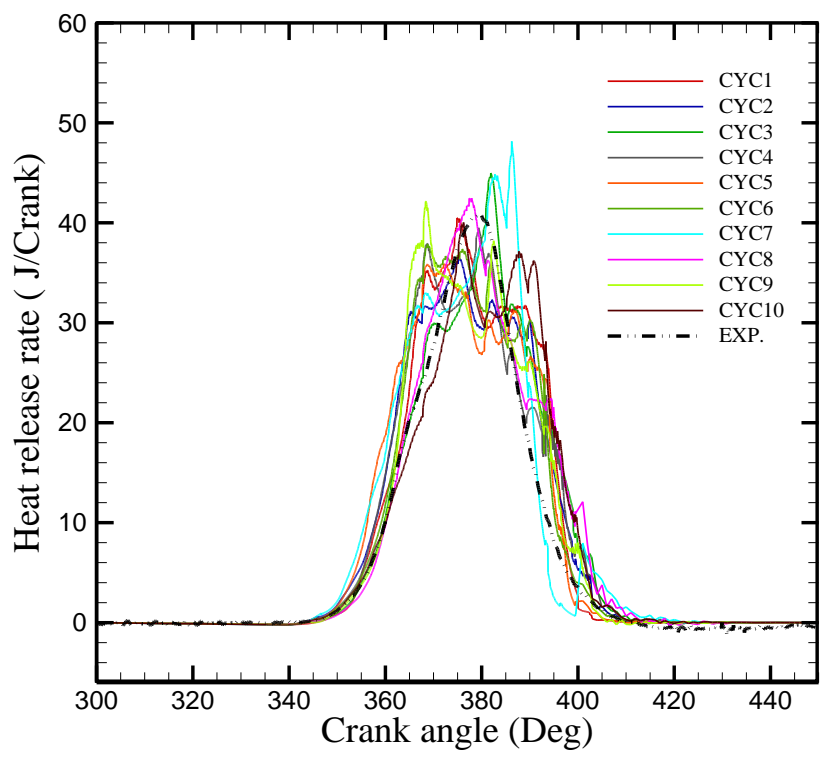

Figure 9. Comparison of predicted heat release rate and computed heat release rate from measured pressure data

Computed heat release rate curves during the combustion process are shown in figure 9. Note that, these results have been processed by applying a low pass filter for clarity of representation. Shown by the chained line is the cycle averaged heat release rate, computed using experimentally measured pressure data. As in the case of in-cylinder pressure, substantial variation of heat release rates can be seen. A general trend of over prediction during the early and latter stages of combustion is apparent, but in the middle phase, the measured and predicted values are comparable. A significant irregularity of heat release is seen in this phase, indicating rigorous flame turbulent interactions.

Despite the successfulness of the present formulation several key points have still to be verified. Mainly, the grid dependency of the model should be evaluated. Sensitivity of the test filters width and the combustion filter width has to be estimated. On the other hand, the model behaviour in different engine operating conditions has also to be thoroughly investigated. Further studies are currently under way to investigate these aspects.

\section{CONCLUDING REMARKS}

FSD based dynamic combustion model and a flame kernel model were implemented in the KIVA-4 code, which was modified to perform LES based engine calculations. Overall flow features have been successfully predicted and larger flow scales have been sufficiently resolved despite the lower mesh resolution and relatively low accurate numerical scheme of KIVA-4 originally developed for RANS. This is believed to be a result of the higher accuracy of the present SGS turbulence model. In addition, global features of flame propagation and reaction rate have been accurately predicted by the present simpler combustion model indicating that LES is a much better tool for simulating engine combustion with an acceptable level of computing cost.

\section{REFERENCES}

1. Haworh, D.C., and Jansen, K., "Large Eddy Simulation on Unstructured deforming meshes: Towards Reciprocating IC Engines," Computers \& Fluids, 29: $493-$ 524, 2000.

2. Sone, K., and Menon, S., "Effect of Subgrid Modelling on the In-Cylinder Unsteady Mixing Process in a Direct Injection Engine," Journal of Engineering for Gas Turbines and Power, 125:435-443, 2003.

3. Huijnen, V., Somers, L.M.T., Baert, R.S.G., and Goey, L.P.H.D., "Validation of the LES Approach in Kiva-3V on a Square Duct Geometry," International Journal for Numerical Methods in Engineering, 64:907-919, 2005.

4. Moureau, V., Barton, I., Angelberger, C., and Poinsot, T., "Towards Large Eddy Simulation in Internal-Combustion Engines: Simulation of a Compressed Tumble Flow," SAE Technical Paper 2004-01-1995, 2004.

5. Goryntsev, D., Sadiki, A., Klein, M., and Janicka, J., "Analysis of Cyclic Variations of Liquid Fuel-Air Mixing Processes in a Realistic DISI IC-Engine Using Large 
Eddy Simulation, International Journal of Heat and Fluid Flow, 31: 845-849, 2010.

6. Solsjö, R., Jangi, M., Tuner, M., and Bai, X., "Large Eddy Simulation of Partially Premixed Combustion in an Internal Combustion Engine," SAE Technical Paper 2012-01-0139, 2012.

7. Goryntsev, D., Sadiki, A., and Janicka, J., "Towards Large Eddy Simulation of Spray Combustion in Direct Injection Spark Ignition Engine," SAE Technical Paper 2011-01-1884, 2011.

8. Heywood, J.B., "Internal Combustion Engine Fundamentals," New York: McGraw-Hill. ISBN: 0-07100499-8.1988.

9. Vermorel, O., Richard, S., Colin, O., Angelberger, C., Benkenida, A. and Veynante, D., "Towards the Understanding of Cyclic Variability in a Spark Ignited Engine Using Multi-Cycle LES," Combustion and Flame, 156:1524-1541, 2009.

10. Poinsot, T., and Veynante, D., "Theoretical and Numerical Combustion," Edwards, ISBN: 1-930217-10-2, 2005 .

11. Fureby, C., and Löfström, C, "Large Eddy Simulations of Bluff Body Stabilized Flames," Twenty-Fifth Symposium (International) on Combustion, 1257 - 1264, 1994.

12. Fureby, C., and Möller, S.I., "Large Eddy Simulation Of Reacting Flows Applied To Bluff Body Stabilized Flames", AIAA Journal, 33: 2339-2347, 1995.

13. Pitsch, H., and Duchamp, "Large-Eddy Simulation of Premixed Turbulent Combustion Using a Level-Set Approach," Proceedings of the Combustion Institute, 29: 2001-2008, 2002.

14. Piana, J., Veynante, D., Candel, S., and Poinsot, T., "Direct Numerical Simulation Analysis of The GEquation In Premixed Combustion," In Direct and Large Eddy Simulation II, Kluwer Academic Publishers, 321-30, 1997.

15. Janicka, J., and Sadiki, A., "Large Eddy Simulation for Turbulent Combustion," Proceedings of the Combustion Institute, 30: 537-547, 2005.

16. Butler, T.D., and O'Rourke, P.J., "A Numerical Method for Two-Dimensional Unsteady Reacting Flows," Sixteenth Symposium (International) on Combustion, 1503-1515. 1997.

17. Colin, O., Ducros, F., Veynante, D. and Poinsot, T., "A Thickened Flame Model for Large Eddy Simulations of Turbulent Premixed Combustion," Physics of Fluids, 12: 1843-1863, 2000.

18. Selle, L., Lartigue, G, Poinsot, T., Koch, R., Schildmacher, K.U., Krebs, W., Prade, B., Kaufmann, P. and Veynante, D., "Compressible Large Eddy Simulation of Turbulent Combustion in Complex Geometry on Unstructured Meshes," Combustion and Flame, 137:489505, 2004.

19. Franzelli, B., Riber, E., Gicquel, L.Y.M. and Poinsot, T., "Large Eddy Simulation of Combustion Instabilities in a Lean Partially Premixed Swirled Flame, Combustion and Flame, 159: 621-637, 2012.
20. Enaux, B., Granet, V., Vermorel, O., Lacour, C., Pera, C., Angelberger, C. and Poinsot, T., " LES Study of Cycle-toCycle Variations in a Spark Ignition Engine, Proceedings of the Combustion Institute, 33: 3115-3122, 2011.

21. Granet, V., Vermorel, O., Lacour, C., Enaux, B., Dugué, V., and Poinsot, T., "Large-Eddy Simulation and Experimental Study of Cycle-To-Cycle Variations of Stable and Unstable Operating Points in a Spark Ignition Engine," Combustion and Flame, 159: 1562-1575, 2012.

22. Wang, G., Boileau, M., Veynante, D., and Truffin, K., "Large Eddy Simulation of a Growing Turbulent Premixed Flame Kernel Using a Dynamic Flame Surface Density Model," Combustion and Flame, 159: 27422754, 2012.

23. Boger, M., Veynante, D., Boughanem, H., and Trouvé, A., "Direct Numerical Simulation Analysis of Flame surface Density Concept for Large Eddy Simulation of Turbulent Premixed Combustion," Proceedings of the Combustion Institute, 27: 917-925, 1998.

24. Sone, K., Patel, N., and Menon, S., "Implementation of Large-Eddy Simulation in to the KIVA code- Part1: Theory and Formulation," Technical Report: CCL-2001008, Georgia Institute of Technology, 2000.

25. Sone, K., Patel, N., and Menon, S., "Implementation of Large-Eddy Simulation in to the KIVA code- Part2: Practicums on Implementation," Technical Report: CCL2001-008, Georgia Institute of Technology, 2000.

26. Ranasinghe, R.A.C.P., "Development of Combustion Models for RANS and LES Applications in SI Engines," PhD Thesis, Mechanical and Manufacturing Engineering Department, Loughborough University, UK, 2013.

27. Calhoon, W.H., "On Subgrid Combustion Modeling for Large Eddy Simulations," PhD Thesis, Georgia Institute of Technology, Atlanta, GA,1996.

28. Boger, M., and Veynante, D., "Large Eddy Simulation of a Turbulent Premixed V-shaped Flame," Proceedings of the Eighth European Turbulent Conference, In Advances in Turbulence VIII, ISBN: 8489925658. 2000.

29. Hawkes, E.R., and Cant, R.S., "Implications of a Flame Surface Density Approach to Large Eddy Simulation of Premixed Turbulent Combustion," Combustion and Flame, 126: 1617-1629, 2001.

30. Herweg, R., and Maly, R.R., "A Fundamental Model for Flame Kernel Formation in S.I. Engines," SAE Technical Paper: 922243, 1992.

31. Richarda, S., Colin, O., Vermorel, O., Benkenida, A., Angelberger, C. and Veynante, D., "Towards Large Eddy Simulation of Combustion in Spark Ignition Engines," Proceedings of the Combustion Institute, 31:3059-3066, 2007.

32. Colin, O., and Truffin, K., “A Spark Ignition Model for Large Eddy Simulation Based on an FSD Transport Equation (ISSIM-LES)," Proceedings of the Combustion Institute, 33:3097-3104, 2000.

33. Dahms, R., Fansler, T.D., Drake, M.C., Kuo, T.W., Lippert, A.M., and Peters, N., "Modeling Ignition Phenomena in Spray-Guided Spark-Ignited Engines", 
Proceedings of the Combustion Institute, 32: 2743-2750, 2009.

34. Dahms, R., Felsch, C., Röhl, O., and Peters, N. "Detailed Chemistry Flamelet Modeling of Mixed-Mode Combustion in Spark-Assisted HCCI Engines", Proceedings of the Combustion Institute, 33: 3023-3030, 2011.

35. Duclos, J.M., and Colin, O., "Arc and Kernel Tracking Ignition Model for 3D Spark Ignition Engine Calculations," COMODIA, 2001.

36. Boudier, P., Henriot, S., Poinsot, T., and Baritaud, T., "A Model for Turbulent Flame Ignition and Propagation in Spark Ignition Engines," Twenty-Fourth Symposium (International) on Combustion, The Combustion Institute, 24: 503-510, 1992.

37. Echekki, T., Poinso,t T., Baritaud, T., and Baurn, M., "Modelling and Simulation of Turbulent Flame Kernel Evolution," In Direct Numerical Simulation for Turbulent Reacting Flows, Editions Technip, ISBN 2-7108-06983:123-146,1996.

38. Charlette, F., Meneveau, C., and Veynante, D., “A PowerLaw Flame Wrinkling Model for LES of Premixed Turbulent Combustion Part I: Non-Dynamic Formulation and Initial Tests," Combustion and Flame, 131: 151-180, 2002.

39. Veynante, D., and Vervisch, L., "Turbulent Combustion Modeling," Progress in Energy and Combustion Science, 28: 193-266, 2002.

40. Ewald, J., and Peters, N., "On Unsteady Premixed Turbulent Burning Velocity Prediction in Internal Combustion Engines," Proceedings of the Combustion Institute, 31: 3051-3058, 2007.

41. Tan, Z., and Reitz, R.D., "Modeling Ignition and Combustion in Spark-ignition Engines Using a Level Set Method," SAE Technical Paper: 2003-01-0722, 2003.

42. Santos, G. R., Lecanu, M., Ducruix, S., Gicquel, O., Iacona, E. and Veynante, D., "Coupled Large Eddy Simulations Of Turbulent Combustion and Radiative Heat Transfer," Combustion and Flame, 152:387-400, 2008.

43. Tabor, G., and Weller, H.G., " Large Eddy Simulation of Premixed Turbulent Combustion Using Flame Surface Wrinkling Model, Flow Turbulence and Combustion, 72:1-28, 2004.

44. Knikker, R., Veynante, D., and Meneveau, C., "A Priory Testing of a Similarity Model for Large Eddy Simulation of Turbulent Premixed Combustion," Proceedings of the Combustion Institute, 29:2105-2111, 2002.

45. Knikker, R., Veynante, D., and Meneveau, C., "A Dynamic Flame Surface Density Model for Large Eddy Simulation of Turbulent Premixed Combustion," Physics of Fluids, 16:.91-94, 2004.

46. Gubba, S.R., Ibrahim, S.S., Malalasekera, W., and Masri, A.R., " LES Modeling of Premixed Deflagrating Flames in a Small-Scale Vented Explosion Chamber with a Series of Solid Obstructions," Combustion Science and Technology, 180:10-11, 2008.

47. Ibrahim, S.S., Gubba, S.R., Masri, A.R. and Malalasekera, W. "Calculation of Explosion Deflagrating

Page 14 of 14
Flames Using a Dynamic Flame Surface Density Model, Journal of Loss Prevention in the Process Industries, 22:258-264, 2009.

48. Gonzalez, R.C., and Woods, R.E., "Digital Image Processing, "2nd Edition, Prentice Hall, 2002.

49. Gülder, Ö.L., "Correlation of Laminar Combustion Data for Alternative SI Engine Fuels." SAE Technical Paper: 841000, 1984.

50. Lund, T.S., Wu, X., and Squires, K.D., "Generation of Turbulent Inflow Data for Spatially-Developing Boundary Layer Simulations," Journal of Computational Physics, 140:233-258, 1998.

51. Rutland, C.J., "Large-eddy Simulation for Internal combustion Engines- A Review," International Journal of Engine Research, 12:1-31, 2011.

52. Yoshikawa, I., Shim, Y., Nada, Y., Tanahashi, M., and Miyauchi, T., "A Dynamic SGS Combustion Model Based on Fractal Characteristics of Turbulent Premixed Flames," Proceedings of the Combustion Institute, In press, 2012.

53. North, G.L. and Santavicca, D.A., "The Fractal Nature of Premixed Turbulent Flames," Combustion Science and Technology, 72: 215-232, 1990.

\section{CONTACT INFORMATION}

Chathura Ranasinghe

e-mail: c.p.ranasinghe@lboro.ac.uk 